\title{
Blocking Temperature and Hysteresis Characteristics of the Decay Products TITANOMAGNETITES
}

\author{
Afremov Leonid Dr \\ Department of Theoretical and Experimental Physics, Far Easter Federal University, Vladivostok, Russian Federation \\ e-mail: afremov.11@dvfu.ru
}

Iliushun Ilia

Department of Theoretical and Experimental Physics, Far Easter Federal University, Vladivostok, Russian Federation e-mail: futted@gmail.com

\begin{abstract}
This work is devoted to the theoretical study of the temperature dependence of the blocking, the coercive force of the spontaneous magnetization and the size of the magnetite core core / shell nanoparticles of magnetite / titanomagnenit. It is shown that the coercive force and the saturation magnetization of a spontaneous increase with increasing volume fraction of magnetite core, which is consistent with experimental data.
\end{abstract}

This work is devoted to the theoretical study of the temperature dependence of the blocking, the coercive force of the spontaneous magnetization and the size of the magnetite core core / shell nanoparticles of magnetite / titanomagnenit. It is shown that the coercive force and the saturation magnetization of a spontaneous increase with increasing volume fraction of magnetite core, which is consistent with experimental data. (Mogensen, 1968). Later Hjelm-Kwist and Ramdohr (см. [1]) showed that separation of magnetite nano are a common feature of the collapse of the titanomagnetite. For example, at the initial stage of

decay titanomagnetites an increase in the coercive force $K_{\sigma}$ and the residual saturation magnetization to saturation

magnetization $l_{\text {rg }} / I_{g}$, some authors [2] show to the small dimensions of the phases. The development process of disintegration increases the size of the phases and a sharp

drop $H_{g}$ и $E_{r g} / L_{g}$.

In this paper, we attempt, within the previously developed model [3.4] two-phase nanoparticles to analyze the effect of decay processes in the hysteresis characteristics titanomagnetite.

\section{INTRODUCTION}

In accordance with the ideas of [5,6], we assume that as a result of the collapse of the primary homogeneous titanomagnetite $\quad \mathrm{Fe}_{3}{ }_{\alpha_{p}} \mathrm{Tt}_{\mathrm{w}_{\mathrm{q}}} \mathrm{O}_{4}$ formed core/shell nanoparticles in which the magnetite core coated titanomagnetite $\left(\mathrm{Fe}_{3-x} \mathrm{Tl}_{2} \mathrm{O}_{4} / \mathrm{Fe}_{2} \mathrm{O}_{4}\right)$ (fig. 1). During decay of the nuclei of magnetite volume $\mathrm{x}$ can be increased, which
Maria Shmykova

Department of Theoretical and Experimental Physics, Far Easter Federal University, Vladivostok, Russian Federation e-mail: cmariaa@mail.ru

will increase the concentration of titanium in the shell volume $s \mathrm{~V}$.

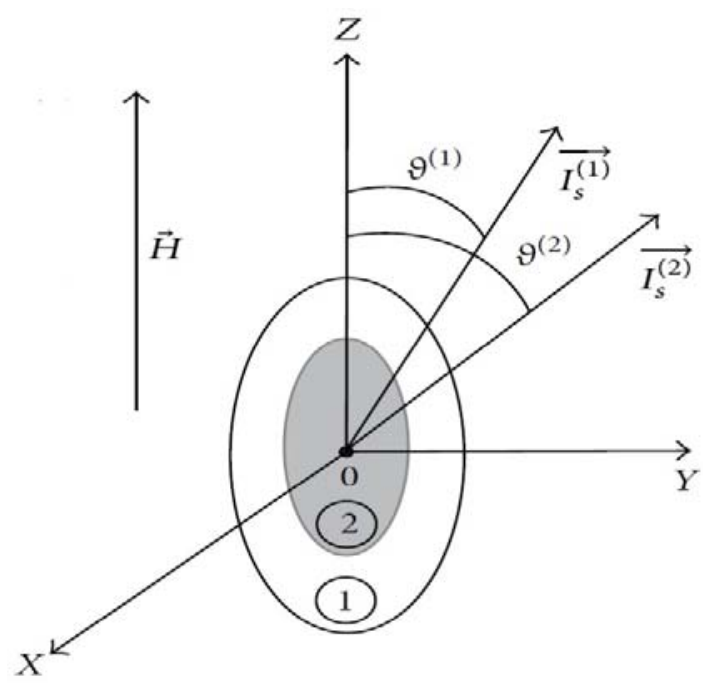

Figure 1. Illustration model core / shell nanoparticles $I_{z}^{51}$ and $E_{s}^{E_{E}}$

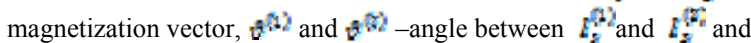
external field $\mathbf{H}$. Uniformly magnetized particle (phase 1) of volume $\mathrm{V}$ ellipsoidal and elongated Q contains uniformly magnetized core (phase 2) with volume $\mathrm{v}=\varepsilon \mathrm{V}$ ellipsoidal inclusion and elongation $\mathrm{q}$

In the process of disintegration of the amount of magnetite cores can be increased, which will lead to an increase in the concentration of titanium in the shell $\mathrm{x}$ volume $\varepsilon \mathrm{V}$. Based on the conservation of the number of titanium atoms in the nanoparticle volume $\mathrm{V}$ and the volume in the shell $(1-\varepsilon) V$ it can establish a link between the initial concentration of titanium $x_{0}$ in nanoparticle with $x$ at any time of the collapse: $W_{T t}=r t V^{2} x_{0}=r t(1-z) \cdot V^{x} x(r t$ - the concentration of molecules titanomagnetites). Thus 


$$
x=\frac{x_{o}}{(1-\varepsilon)}
$$

From (1) should be a limit on the maximum value of the relative volume of magnetite core $\varepsilon_{m}=1-x_{0}$ appropriate $x=1$.

The described process of decay can lead to a change in hysteresis characteristics of nanoparticles (coercive force

$$
l(t)=c\left[\left(n_{1}(t)-n_{3}(t)\right)\left((1-\varepsilon) l_{s}^{(1)}+\varepsilon l_{s}^{(2)}\right)+\left(n_{2}(t)-n_{4}(t)\right)\left|(1-\varepsilon) l_{s}^{(1)}-\varepsilon l_{s}^{(2)}\right|\right]
$$

Where $n(t)-\left\{n_{1}(t) n_{2}(t) n_{2}(t), n_{4}(t)\right\} \quad$ vector populations of the states of two-phase nanoparticles with components $n_{0}(t)$ can be regarded as the probability of finding nanocha $\neg$ stitsy in one of four conditions: first

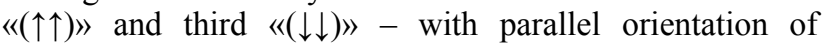

$$
\frac{d n_{i}(t)}{d t}=\sum_{k \neq i}^{4}\left(-W_{i k} n_{i}(t)+W_{i k} n_{k}(t)\right), n_{i}(0)=n_{0 i}
$$

Where $W_{t k}=f_{0} \exp \left(-E_{i k} / k_{\mathrm{g}} T\right)$ - elements of the matrix of transition probabilities $i-$ th equilibrium in $k$ - th,

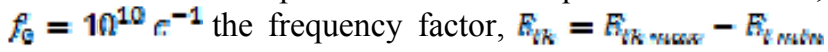
- energy barriers separating $i-$ th и $k-$ th equilibrium states ( $E_{t k \text { max }}$ - the smallest of the maximum values of power sharing $i-$ th и $k$ - the states, $E_{t \text { mon }}-$ energy $i-$ th equilibrium). A detailed calculation of energy barriers presented in the book [4].

\section{NANOPARTICLES BLOCKING TEMPERATURE TITANOMAGNETITE / MAGNETITE}

Since the eigenvalues $W_{t k}$ determine the inverse times of transition from one state to another $\tau_{n}$, to estimate the time of the transition to the equilibrium state will use the maximum of them: $\tau=\left(\tau_{n}\right)_{\text {maw }}$. We define the temperature block $T_{5}$ as the temperature at which the relaxation time of $\tau=1 \tau$.

The simulation results of temperature block $T_{B}$ cspherical nanoparticles $\mathrm{Fe}_{2: 0} \mathrm{Tt}_{\mathrm{Q}_{2}} \mathrm{O}_{4} / \mathrm{Fe}_{3} \mathrm{O}_{4}$ size $1 \mathrm{CO} \mathrm{nm}$ from radius magnetite core titanomagnetite, which can be considered as a measure of decay titanomagnetites, showed on fig. 2. The figure shows that the temperature of blocking virtually unchanged until $\mathrm{m} 20 \mathrm{~nm}$ and rapidly increases with the size of the magnetite core. magnetic moments of the two phases, the second «( $\uparrow \downarrow) »$ and fourth «( $\downarrow \uparrow) »-$ with antiparallel magnetic moments of the phase (see. Fig. 1). Change the vector population of two-phase nanoparticles can be described by the following equation [4]:

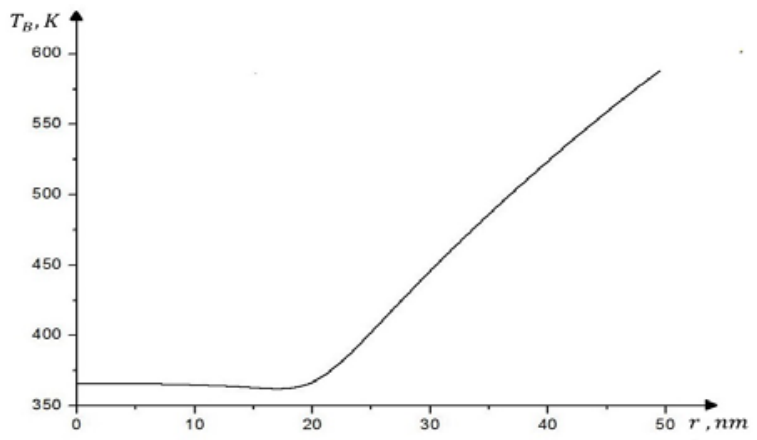

Figure 2. Temperature dependence of the blocking nanoparticles from the size of magnetite core $r$.

This behavior is determined by the dependence $\mathrm{T} B$ potential barrier height of the size of the nucleus (see. Fig. 3 ). Fig. 3 shows the potential barriers, defining the reversal nanoparticles being in the first state. It can be done in two ways: by the magnetic moments of the coup or phase synchronous magnetic reversal of the magnetic moment of the nanoparticle. In the first case, a transition occurs from the first state «( $\uparrow \uparrow) »$ second «( $\uparrow \downarrow) »-$ through the barrier $E_{12}$ (or fourth «( $\downarrow \uparrow) »-$ through the barrier $E_{14}$ ), and then the third «( $\downarrow \downarrow) »-$ through the barrier $E_{23}$ or $E_{48}$. In the second - from the first $«(\uparrow \uparrow) »$ to third «( $(\downarrow))$ - through the barrier $E_{18}$. From fig. 3 that for nanoparticles from simulated all of the above transition occurs transitions from the first to the fourth, and then a third state. Moreover, the potential barrier $E_{14}$, defining the reversal of nanoparticles c $r<20 \mathrm{HM}$, practically independent of size of the nucleus, whereas the magnetization reversal nanoparticles $r \geqslant 20 \mathrm{~nm}$ controlled barrier $E_{43}$, which increases rapidly with increasing $r$. 


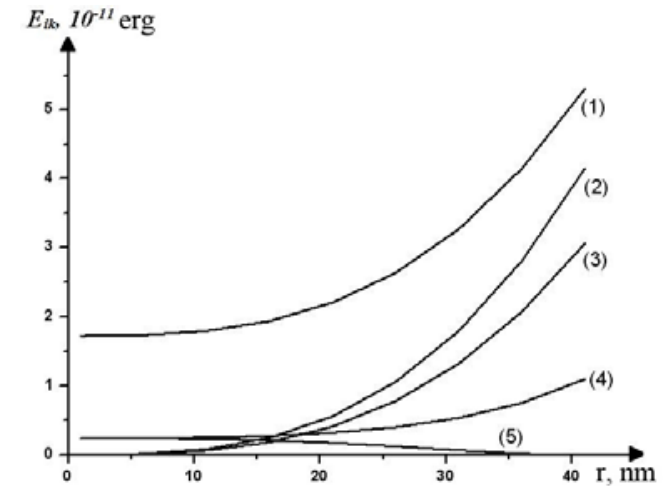

Figure 3. The dependence of the potential barriers $E_{i k}$ on the size of the magnetite core r. curves: $1-\boldsymbol{E}_{1 \mathrm{z}}, 2-\boldsymbol{E}_{\mathbf{1 z}}, 3-\boldsymbol{E}_{\mathbf{1 z}}, 4-\boldsymbol{E}_{\mathbf{1}, 1}, 5-\boldsymbol{E}_{\mathbf{z z}}$.

Thus, as expected, with increasing magnetite core temperature rise occurs blocking nanoparticles. Nanoparticles with a high content of magnetite are more stable against thermal stress, which is consistent with experimental data $[6,7]$.

\section{THE COERCIVE FORCE AND SATURATION MAGNETIZATION}

The coercive force $H_{\varepsilon}$ and saturation magnetization $I_{g}$ the system of nanoparticles titanomagnetites $\mathrm{Fe}_{2.8} \mathrm{Ti}_{\mathrm{Q}_{2}} \mathrm{O}_{4} / \mathrm{Fe}_{3} \mathrm{O}_{4}$ o determined from the hysteresis loop, which built by the relations (2) - (3). Naturally, $H_{c}$ and $I_{s}$ nanoparticles increase with increasing magnetite nanoparticle (see. Fig.4). It should be noted the area in which the growth of magnetite core has no significant effect on the coercive force of the system.

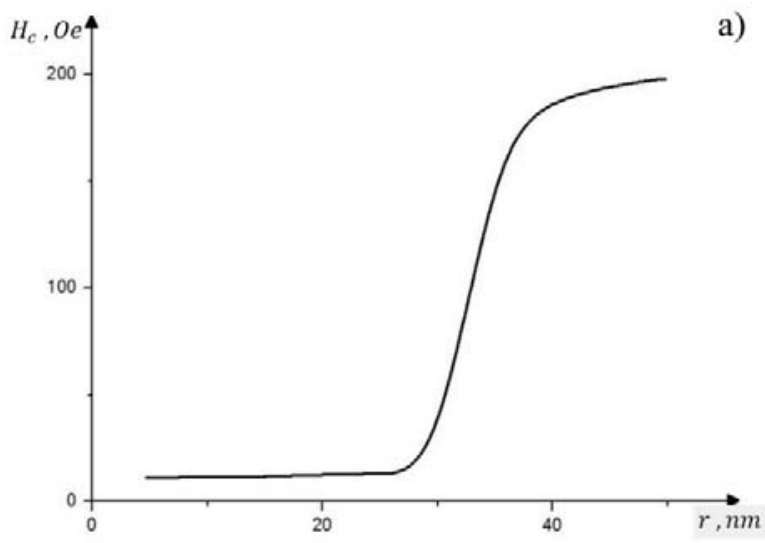

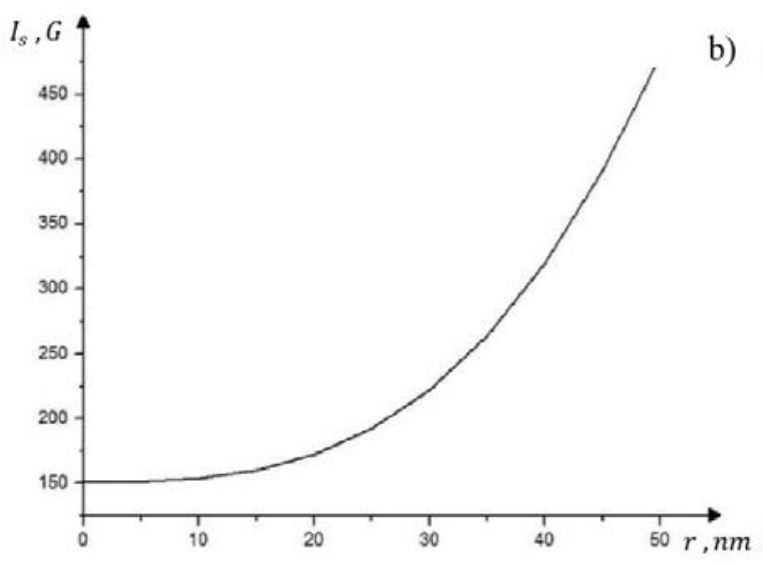

Figure. 4. The dependence of the saturation magnetization Is (a) and the coercive force Hc (b) the radius of the nanoparticles $F_{\mathrm{z}_{\mathrm{g}}} T_{\mathrm{g}, \mathrm{g}} \mathrm{O}_{4} / \mathrm{F}_{\mathrm{g}} \mathrm{O}_{4}$.

Obviously, this is due to the low values of the potential barriers $E_{14}$ и $E_{43}$ when the size of the magnetite core $r<30 \mathrm{~nm}$, which do not exceed $10^{-11}$ Erg (see. Fig. 3). The sharp increase in coercive force of up to a maximum value corresponding to $H_{c}$ Magnetite nanoparticles with an increase in the core $r \geqslant 30 \mathrm{~nm}$, associated with a significant ( 5 - fold) increase in potential barriers (see. fig. 3 ). Obviously, a slight change in potential barriers in the $20<r<30 \mathrm{~nm}$ can not neutralize the thermal fluctuations of the magnetic moments of the phase nanoparticles, which determines the weak growth of the coercive force in the above area. The dependence of the coercive force of the size of the magnetite core is in good agreement with the experimental results $[2,6]$.

\section{CONCLUSION}

In the model core / shell nanoparticles [3] studied the temperature dependence of the blocking, the coercive and the saturation magnetization of the size of the magnetite core. It is shown that an increase in the particle core temperature of blocking does not change up to a value of 20 $\mathrm{nm}$, and then begins to increase rapidly. The coercive force and spontaneous saturation magnetization increases with increasing volume fraction of magnetite core. These results are in good agreement with the experimental data $[2,6]$.

\section{ACKNOWLEDGMENT}

Work supported by Ministry of Education and Science, project № 559.2014.

\section{REFERENCES}

[1] Stacey, F.D., Banerjee, S.K. "The physical principles of the rock magnetism". Amsterdam. Elsevier. 1974. 195 pp.

[2] Artemova T.G., Gapaeev A.K. "On the decay of solid solutions in the magnetite-ulvospinel” Bulets. USSR. Vol. Ears Physics. 1988. № 12. Pp. 82-87.

[3] Afremov L. L., Ilyushin I. G. "Effect of mechanical stress on magnetic states and hysteresis characteristics of a two-phase nanoparticles system", Journal of Nanomaterials Volume 2013, 2013, Article number 687613, DOI: 10.1155/2013/687613 
[4] Afremov L. L., Panov A. V. "Theory of Magnetization of Two-Phase Superparamagnetic Particles: II. Modeling of Magnetization Processes." The Physics of Metals and Metallography, 1996, vol. 82, No. 5, pp. 7-23.

[5] Kakol Z., J. Sabol, J.M. "Honig, Magnetic anisotropy of titanomagnetires Fe_,Ti,O4, 0 x 0.55.” Phys. Rev. B 43, (1991), pp.1.

[6] Pearce C.I., C.M.B. Henderson, R.A.D. Pattrick, G. van der Laan, D.J. "Vaughan Direct determination of cation site occupancies in natural ferrite spinels by L2,3 X-ray absorption spectroscopy and Xray magnetic circular dichroism", American Mineralogist, Volume 91, Issue 5-6, (2006), Pages 880-893

[7] Pearce C.I. et al. "Synthesis and properties of titanomagnetite (Fe3-xTixO4) nanoparticles: A tunable solid-state $\mathrm{Fe}(\mathrm{II} / \mathrm{III})$ redox system", Journal of Colloid and Interface Science 387,2012, pp. 2 